\title{
Measurement of thermal expansion coefficient of poly-Si using microgauge sensors
}

\author{
Jung-Hun Chae, Jae-Youl Lee, Sang-Won Kang \\ Department of Materials Science \& Engineering, Korea Advanced Institute of Science and Technology, \\ 305-701, Kusong-dong, Yusong-gu, Taejon, Korea
}

\begin{abstract}
Thermal expansion coefficient of heavily doped LPCVD polycrystalline (poly-Si) thin film was extracted by microgauge sensors. When electrical power was applied to the microgauge, it was heated up and thermal expansion occurred. From the relation between applied current and measured displacement at the microgauge, thermal expansion coefficient of thin film was extracted. The results revealed a value of $2.9 \times 10^{-6} / \mathrm{K}$ of thermal expansion coefficient of highly doped poly-Si thin films with standard deviation of $0.24 \times 10^{-6} / \mathrm{K}$.
\end{abstract}

Keywords: thermal expansion coefficient, microgauge, MEMS, thin film

\section{INTRODUCTION}

Poly-Si is frequently used for active layers in many applications in the field of microelectromechanical systems (MEMS) based on Si micromaching. Especially, the proper design of thermally driven microactuators requires a sound understanding of the process dependence of basic material properties like thermal expansion coefficient ${ }^{1-3}$. Also, optical measurement of intrinsic stress of thin film originated from deposition process requires exact values of thermal expansion coefficient of deposited thin film ${ }^{4-5}$. Therefore thermal expansion coefficient for phosphorus doped poly-Si thin film was studied using microgauge method. In this paper, a micromachined poly-Si microgauge structure for measurement of thermal expansion coefficient of thin film is presented. Microgauge structures have been used for measuring intrinsic stress of the thin film ${ }^{6}$. In this work, thermal expansion of microgauges due to Joule heating were used to extract thermal expansion coefficient of poly-Si thin film. Thermal expansion coefficient of poly-Si thin films were extracted from the relation between calculated average temperature and measured displacement at the microgauge. In microgauge equation, thermal expansion coefficient of thin film is represented as a function of its average temperature, design parameters and measured displacement at the Vernier site. Therefore, by measuring the displacement and calculating average temperature of the microgauge, thermal expansion coefficient of thin film can be calculated. The average temperature of the microgauge, when current was applied, was calculated from heat conduction equation with simple modeling. Microgauge method has some advantages compared with other reported optical techniques to measure thermal expansion coefficient of thin films. Mechanical properties of substrate like Elastic modulus, thermal expansion coefficient is not needed in microgauge method because all measured parts of the thin film are free standing above substrate.

\section{DEVICE STRUCTURE}

The schematic illustration of the microgauge is shown in Fig. 1, in which the idea is to magnify the tiny displacement caused by thermal expansion of test beam. Dark parts are unmovable anchors and clear parts are movable free standing structures.

When electric power is applied to the microgauge, it heats up and thermal expansion of the test beam occurs due to Joule heating effect. The movement of the test beam due to thermal expansion cause the movement of the slope beam and creates tiny rotations. The indicator beam magnifies the slope and a large displacement is generated at the site of the Vernier gauge. The displacement at the Vernier gauge is measured with optical microscope equipped with CCD camera and color image printer. 


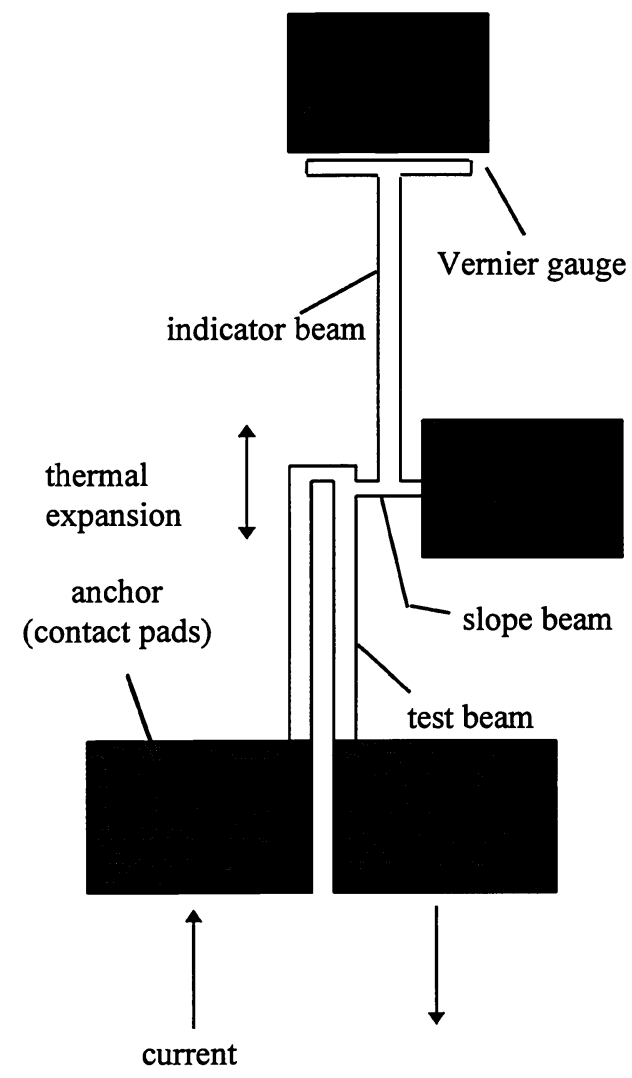

Fig. 1. Schematic illustration of microgauge structure
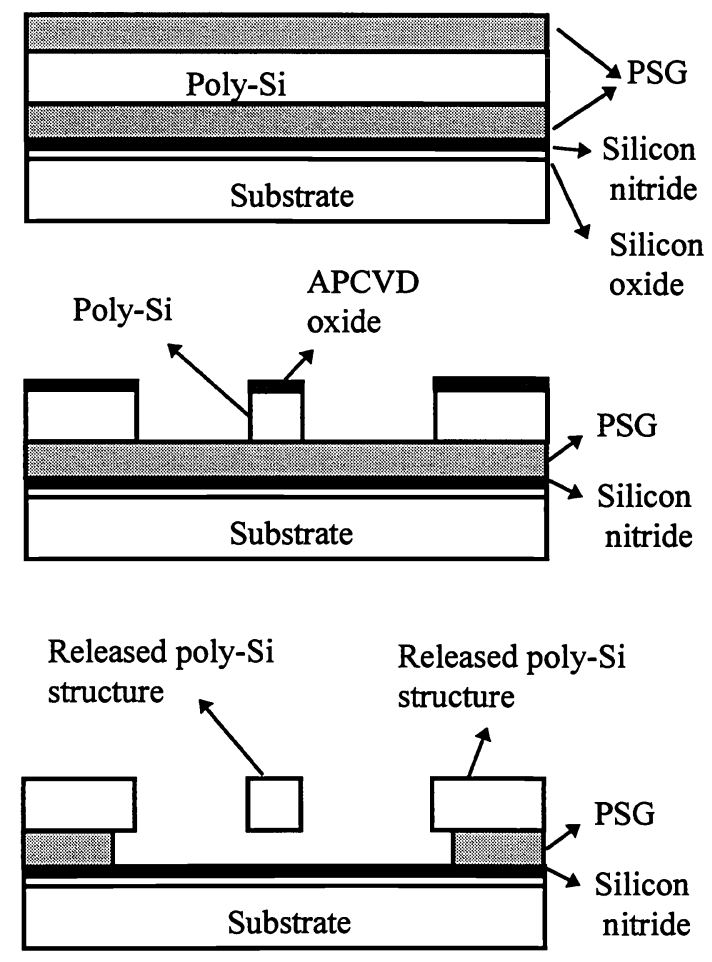

Fig. 2. Schematic sequence illustration of device

\section{FABRICATION}

The microgauge fabrication process is shown in Fig. 2. For the fabrication of the structures, a standard surface micromachining technology was used. The fabrication process for the device started with 4 inch, n-type (100) silicon wafers. 1000A thick thermal silicon oxide layer was grown which served as a stress buffer layer for silicon nitride layer. $1000 \mathrm{~A}$ thick silicon nitride layer was deposited to electrically isolate devices from substrate. $2 \mu \mathrm{m}$ thick LPCVD phosphosilicate glass (PSG) layer was deposited. This PSG layer was used both as the sacrificial layer for the device structure and for the phosphorus diffusion source. After the PSG deposition, $3 \mu \mathrm{m}$ thick, poly-Si layer was deposited for the device material. Again $2 \mu \mathrm{m}$ thick PSG layer was deposited on the poly-Si layer for phosphorus diffusion source. This step was followed by a high temperature phosphorus diffusion step at $1000^{\circ} \mathrm{C}$ for 10 hours which also anneals out intrinsic stress in the deposited poly-Si layer. Upper PSG layer was stripped out in buffered oxide etch (BOE) solution. 6000A thick APCVD undoped oxide layer was deposited which served as a hard mask for dry etching poly-Si layer. Following, a photolithographic step was used to define the device structures on the APCVD undoped oxide layer. Next, 6000A of APCVD oxide layer was patterned by dry etching. $3 \mu \mathrm{m}$ thick poly-Si layer was dry etched with APCVD oxide hard mask. In this dry etching process, all device structures were defined. After dry etching device structures, APCVD oxide layer was removed by dry etching. 5000A thick Aluminum metallization followed and second photolithographic process was used to define the contact pads. Time controlled PSG etch in BOE solution made the device free stand. The PSG etch was stopped when the devices are free stand completely. But device supports for the poly-Si device structures were only slightly undercut because of their larger size. For the last step, $25.4 \mu \mathrm{m}$ (1-mil) thick Aluminum wire was bonded between contact pads of the device structures and copper lead frames. 


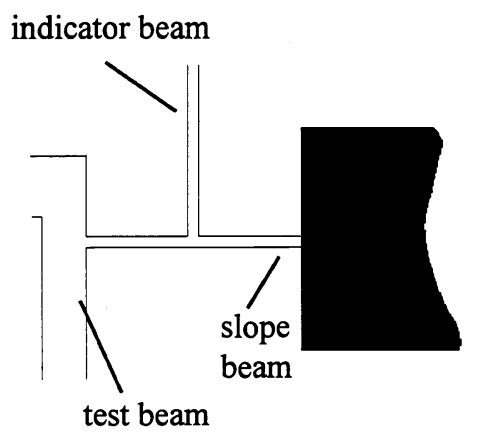

(a)

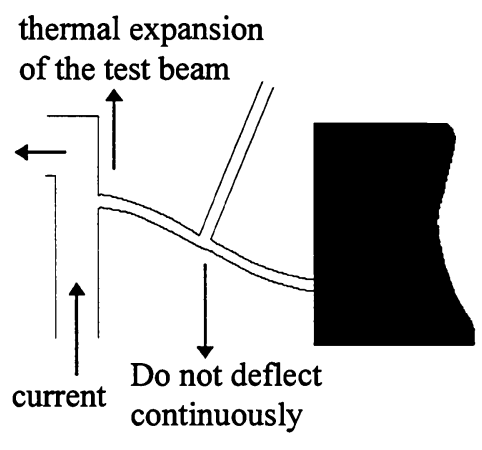

(b)

Fig. 3. Deflection of the slope beam due to thermal expansion of the test beam.

(a) No applied current.

(b) With applied current. The slope beam does not deflect continuously as beam theory expects due to the presence of the indicator beam.

\section{THEORY}

\section{MECHANICAL THEORY}

Measured displacement of the Vernier site of the microgauge, $\delta$, and the amount of expansion of the test beam can be related by beam analysis of the slope beam. Fig. 3. is a view of the slope beam, in which one may observe the fixed-fixed boundary conditions of the ends of the test beams and a small displacement due to thermal expansion of the test beam. This displacement creates tiny angular deflections along the slope beam. Linwei Lin $^{6}$ modeled this situation simply and expressed the relation between strain at the test beam and measured displacement at the Vernier gauge as

$$
\varepsilon=\frac{2 L_{s} \delta}{3 L_{i} L_{f}}
$$

where $L_{s}$ is the length of the slope beam, $\delta$ is measured displacement at the microgauge, $L_{i}$ is the length of the indicator beam, $L_{t}$ is the length of the test beam. The slope beam can not deflect continuously as beam theory due to the presence of the indicator beam. $c_{\mathrm{f}}$ is a correction factor for the slope beam and can be derived as ${ }^{6}$

$$
C_{f}=\frac{1-d^{2}}{1-d^{3}}
$$

where $d$ is the ratio of the width of the indicator beam, $w_{i}$, over the length of the slope beam, $L_{s}$. The strain at the test beam due to thermal expansion can be represented as

$$
\varepsilon=\alpha \Delta T=\alpha\left(T_{a v r}-T_{s}\right)
$$

where $\alpha$ is thermal expansion coefficient of thin film, $\mathrm{T}_{\mathrm{avr}}$ is average temperature of the microgauge when current is applied, $T_{s}$ is the temperature of the substrate. Rewriting eq. (1) and eq. (3), thermal expansion coefficient of the thin film can be derived as 


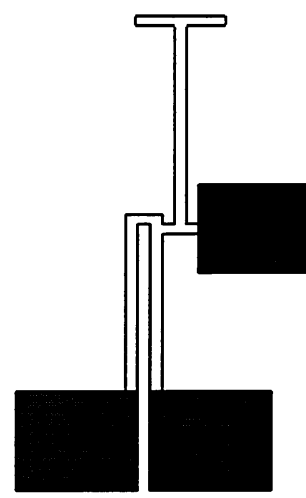

(a)

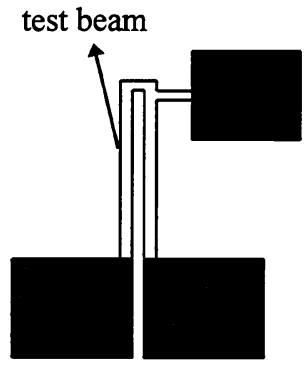

(b)

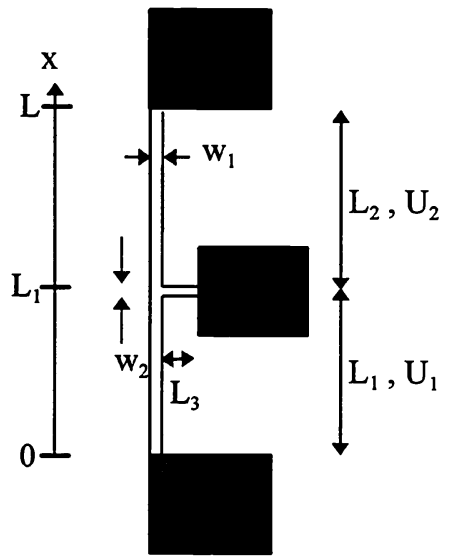

(c)

Fig. 4. Simple modeling of the microgauge as a T-shaped microgauge.

(a) Initial feature of the microgauge.

(b) The indicator beam can be neglected in heat conduction equation because it is floated with respect to heat flow.

(c) T-shaped microgauge is equivalent to the microgauge with respect to the heat conduction equation.

$$
\alpha=\frac{2 L_{s} \delta}{3 L_{i} L_{t} C_{f}\left(T_{a v r}-T_{s}\right)}
$$

\section{THERMOELECTRICAL THEORY}

According to the equation (4), it is necessary to know the average temperature of the microgauge when the current was applied in order to calculate the thermal expansion coefficient of thin film. The average temperature of the microgauge was calculated by the following sequences.

First, the temperature profiles of the microgauge along the test beam length was induced from differential heat conduction equation. In this step, the microgauge was modeled as T-shaped microgauge as shown in Fig. 4.

Second, the temperature profiles were integrated along the test beam length, and then divided by its total length, resulted in average temperature of the microgauge.

Fig. 4. (c) shows $T$-shaped microgauge. $U_{1}$ is the temperature profile along the bridge region $0<x<L_{1}, U_{2}$ is the temperature profile region $\mathrm{L}_{1}<\mathrm{x}<\mathrm{L}$.

If the power is applied to the microgauge, there are three major heat dissipation mechanisms. First, heat is directly conducted through the beam itself to the contact pads of the microgauge and then to the substrate. Second, heat is dissipated by conductive and convective mechanism through the air. Third, heat is dissipated to the environment in the form of infrared radiation. If the microgauge is driven in vacuum chamber, in this experiment $10 \mathrm{mtorr}$, heat loss through the air convection or conduction is negligible compared with heat generated in the microgauge by Joule heating. If the average temperature of the microgauge does not exceed $600^{\circ} \mathrm{C}$, in this experiment $200^{\circ} \mathrm{C}$, heat loss by radiation is also negligible. The amount of heat loss by convection and conduction through the air and radiation loss was calculated as Mastrangelo ${ }^{7}$ did. 

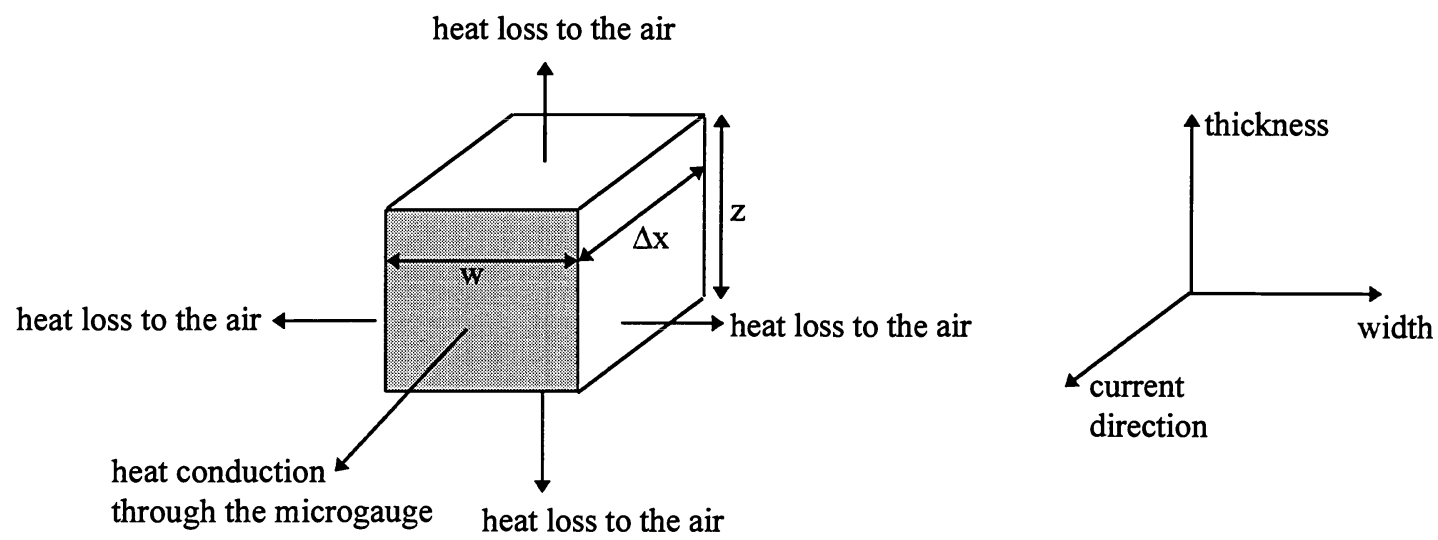

Fig. 5. Differential element of the microgauge structure.

In order to know the amount of heat generated by Joule heating effect, temperature characteristics of resistivity of microgauge material, poly-Si, is needed because the resistivity of thin film and the amount of Joule heating are affecting each other. In particular, for heavily doped poly-Si, the temperature characteristics of resistivity obey the linear relationship as

$\rho=\rho_{0}\left[1+\xi\left(T-T_{0}\right)\right]$

where $\xi$ is the temperature coefficient of resistivity, $\rho_{0}$ is the resistivity of poly-Si thin film at temperature $T_{0}$. This relationship will be verified by some sets of experiments in this paper.

If we consider the differential element of the T-shaped microgauge of width $w$, thickness $z$ and length $\Delta x$ shown in Fig. 5 . the heat valance of the differential element is given by

$Q_{\text {total }}=P_{g}+Q_{\text {conduction }}$

where $Q_{\text {total }}$ is the net rate of change of the internal energy of the element, $P_{g}$ is the power generated inside the element and $Q_{\text {conduction }}$ is the heat loss by thermal conduction of the microgauge. In eq. (6), $Q_{\text {total }}$ is given

$Q_{\text {total }}=\rho_{m} c w z \Delta x\left(\frac{\partial u}{\partial t}\right)$

where $\mathrm{c}$ and $\rho_{\mathrm{m}}$ are the specific heat and density of the microgauge material, respectively, and $\mathrm{u}$ is the average temperature of the element. The power generation term $\mathrm{P}_{\mathrm{g}}$ is

$P_{g}=J^{2} \rho(u) w t \Delta x=J^{2} \rho_{0} w t \Delta x\left[1+\xi\left(u-T_{0}\right)\right]$

where $J$ is the current density flowing through the element, $\rho_{0}$ is its resistivity at $T_{0}$ and $\xi$ is temperature coefficient of the resistivity (TCR). The conductive component is

$Q_{\text {conduction }}=-k W z\left(\left.\frac{\partial u}{\partial x}\right|_{x}-\left.\frac{\partial u}{\partial x}\right|_{x+\Delta x}\right)$

where $\mathrm{k}$ is thermal conductivity of the beam material. 


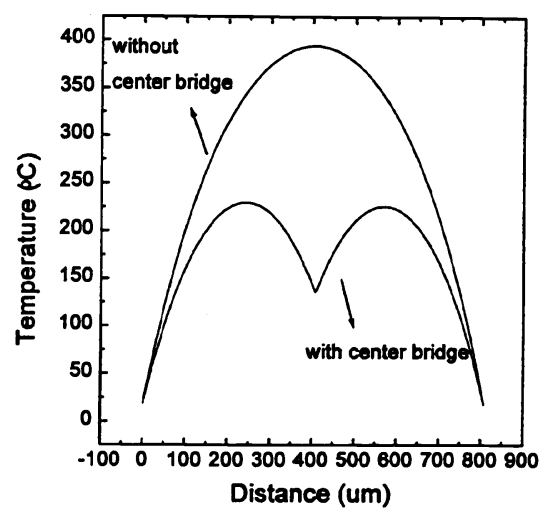

Fig. 6. Temperature profiles of microgauge along test beam length. $\mathrm{L}=800 \mu \mathrm{m}, \mathrm{L}_{1}=407.5 \mu \mathrm{m}, \mathrm{L}_{3}=20 \mu \mathrm{m}, \mathrm{z}=3 \mu \mathrm{m}$ and applied current is 500uA.

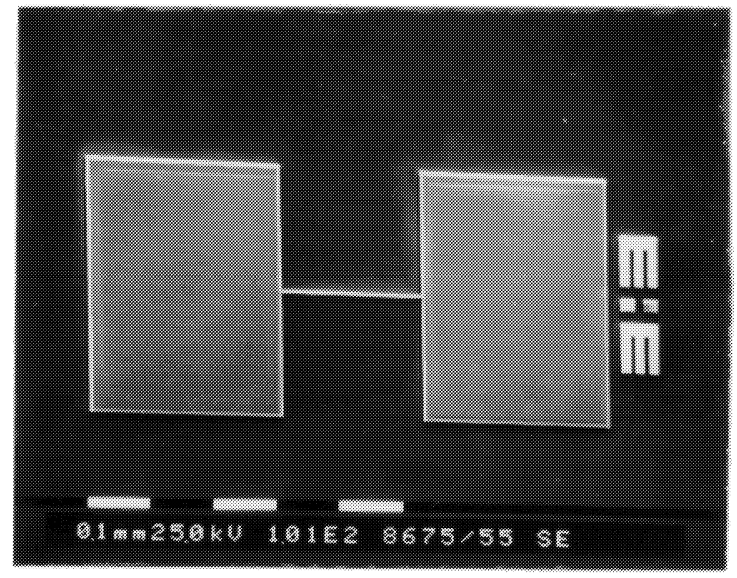

Fig. 7. SEM image of poly-Si microbridge. Bridge length $=300 \mu \mathrm{m}$, width $=3 \mu \mathrm{m}$, thickness $=3 \mu \mathrm{m}$.

Rewriting eq. (6) - eq. (9)

$c \rho_{m} W z \Delta x\left(\frac{\partial u}{\partial t}\right)=J^{2} \rho_{0} W z \Delta x\left[1+\xi\left(u-T_{0}\right)\right]-k w z\left(\left.\frac{\partial u}{\partial x}\right|_{x}-\left.\frac{\partial u}{\partial x}\right|_{x+\Delta x}\right)$

For steady state condition, eq. (10) can yields the linear second-order partial differential equation

$\frac{\partial^{2} u_{i}}{\partial x}+\frac{J^{2} \rho_{0} \xi}{k} u_{i}+\frac{J^{2} \rho_{0}}{k}\left[1-\xi T_{0}\right]=0 \quad(i=1,2)$

eq. (11) is solved subject to the boundary and initial conditions

$u_{1}[x=0]=u_{2}[x=L]=T_{s}$

$-\left.k W_{1} z\left(\frac{\partial u_{1}}{\partial x}\right)\right|_{x=L_{1}}-\left.k W_{1} z\left(\frac{\partial u_{2}}{\partial x}\right)\right|_{x=L_{2}}=\frac{k W_{2} z}{L_{3}}\left(u_{1}\left[x=L_{1}\right]-T_{s}\right)$

We assumed in eq. (12) that the temperature at the microgauge anchors $(x=0, x=L)$ is identical to that of the substrate $T_{s}$. According to Mastrangelo's calculation ${ }^{7}$, this condition is true as long as the power conducted through the anchors does not exceeds a few milliwatts. Second assumption in eq. (13) is that the amount of heat conducted away through the slope beam is same as the sum of the heat conducted from region $0<\mathrm{x}<\mathrm{L}_{1}$ and $\mathrm{L}_{1}<\mathrm{x}<\mathrm{L}$. With the aid of boundary and initial conditions, eq. (11) results in the temperature profiles of the microgauge in region $0<x<L_{1}$ and $L_{1}<x<L$ respectively. Integrating the temperature profile along the microgauge length and divide it by its total length results in average temperature of the microgauge. Fig. 6 . shows an example of the steady state temperature profiles of eq. (11) when $\mathrm{L}=800 \mathrm{um}, \mathrm{L}_{1}=407.5 \mathrm{um}$, $\mathrm{L}_{3}=20 \mathrm{um}, \mathrm{z}=3 \mathrm{um}$ and applied current is $500 \mathrm{uA}$ and when there is no slope beam (center beam) at the same condition. 


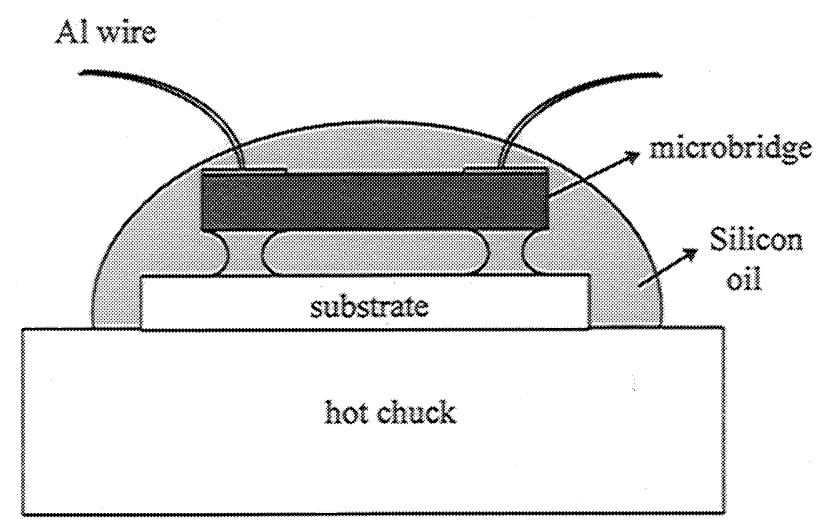

Fig. 8. Oil-bath method for measuring resistivity of microbridge.

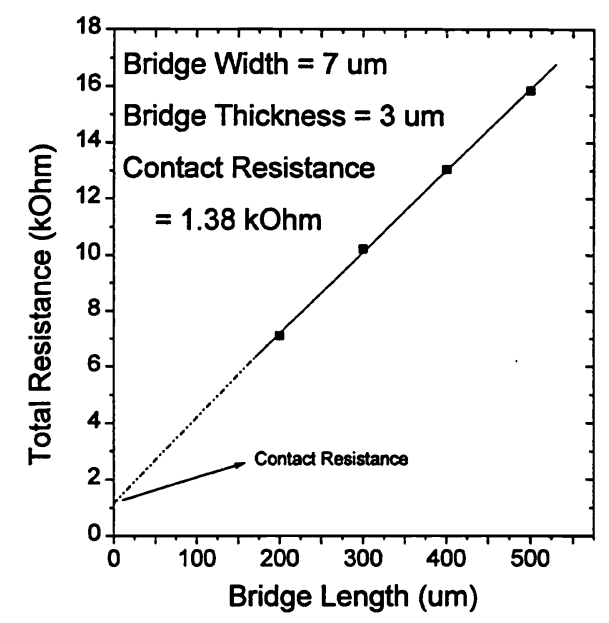

Fig. 9. Total bridge resistance as a function of

bridge length

\section{EXPERIMENTS AND RESULTS}

\section{TEMPERATURE CHARACTERISTICS OF RESISTIVITY OF POLY-SI THIN FILM}

The microgauge heat equation (11) is valid only if the temperature dependence of the poly-Si resistivity obeys the linear relationship in eq. (5). To explore this relationship, we experimented with microbridges shown in Fig. 7. Because fabricated at the same process, the microgauge and the microbridges had same electrical properties each other. the dimensions of the microbridges were determined at the design process, the resistivity as a function of temperature of poly-Si thin film could be obtained from measuring the resistance of the microbridge as a function of temperature. The temperature characteristics of resistivity was measured by oil-bath method as Fig. 8. The microbridge was immersed in insulating silicon oil. The oil acted as a heat exchanger, holding the microbridge temperature at a series of values of $T_{\text {oil }}$ that were adjusted by a hot chuck used as an external heat source. This oil-bath method ensured uniform temperature for entire parts of the microbridge. Hot chuck was used in order to heat the microbridge and silicon oil from room temperature up to $200^{\circ} \mathrm{C}$. Measuring the resistance of the microbridge at any temperature resulted in the resistivity of the poly-Si thin film because we know the dimensions of the microbridge. Small measuring current less than $10 \mathrm{uA}$ was applied to the microbridge in order to minimize Joule heating effect of the microbridge. If the measuring current was large, the temperature of the microbridge could be different from that of the silicon oil because of Joule heating effect. Measuring current was applied by 4145B HP Semiconductor Parameter Analyzer. But it is important to note that the measured resistance of the microbridge was consist of two factors. One was the resistance of the microbridge itself and the other was the contact resistance of the microbridge system. So it was needed to know the contact resistance of the microbridge in order to measure the measure the resistivity of the poly-Si thin film exactly. We used poly-Si microbridges of various sizes to measure the contact resistance of the poly-Si. These microbridges had the same thickness (z), same width (w) but had different lengths (L). Fig. 9. shows measured resistance of the microbridges as a function of total microbridge length. Extrapolating the linear line in Fig. 9. to the point where the microbridge length equals zero, y-intersection point, we can obtain contact resistance of the microbridge system at that point. That y-intersection point means that the resistance of the microbridge when the bridge length equals zero, hence contact resistance of the microbridge. Considering contact resistance of the microbridge, we obtained temperature characteristics of resistivity as shown in Fig. 10. This relationship could be fitted linearly as equation (5) and its parameter values were as below in temperature range from room temperature up to $200^{\circ} \mathrm{C}$. 


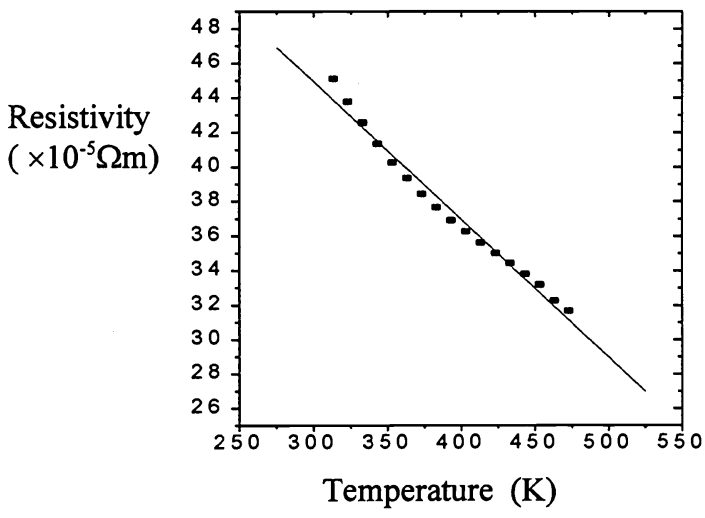

Fig. 10. Temperature coefficient of resistivity of poly-Si

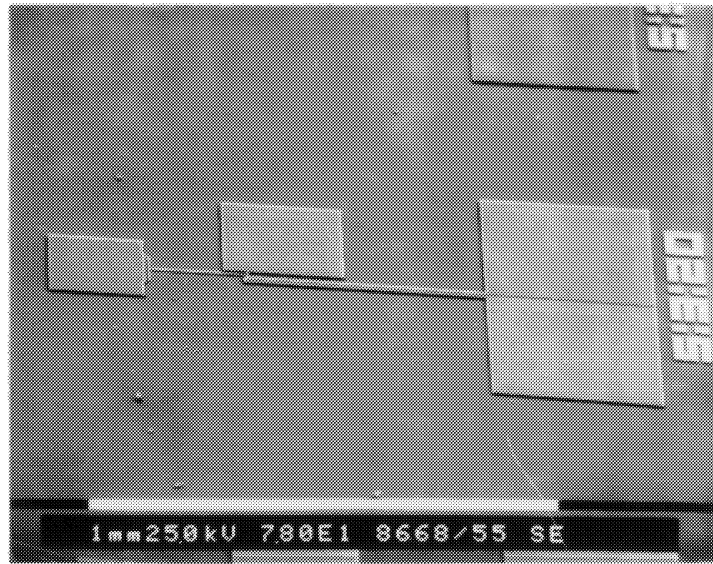

Fig. 11. SEM image of microgauge.

thin film.

$\rho_{0}=5.7 \times 10^{-4}-6.4 \times 10^{-4}[\Omega \mathrm{m}]$ at $\mathrm{T}_{0}=19^{\circ} \mathrm{C}$

$\xi=-1.74 \times 10^{-3} /{ }^{\circ} \mathrm{C}$

From this experiment, $\rho_{0}$ values showed some deviations from sample to sample. But TCR, $\xi$, value was always measured as constant value, $-1.74 \times 10^{-3} /{ }^{\circ} \mathrm{C}$, independent of samples.

\section{THERMAL EXPANSION COEFFICIENT OF POLY-SI THIN FILM}

Thermal expansion coefficient of poly-Si thin film was extracted with microgauge shown in Fig. 11. The microgauges were driven in $10 \mathrm{mtorr}$ vacuum chamber to avoid heat loss to the air. Current was applied to the microgauge via copper lead frame, Al wire and then contact pads of the microgauge by 4145B HP Semiconductor Parameter Analyzer. The displacement at the microgauge was measured with optical microscope equipped with CCD camera and color image printer. Fig. 12. shows the Vernier site of the microgauge when no current was applied and current was applied, indicating some displacement at the Vernier gauge due to applied current. The displacement of the microgauge due to the applied current was measured from the difference between its last and initial positions of the Vernier gauge. The average temperature of the microgauge when current was applied was calculated from eq. (11). Thermal expansion coefficient was calculated from eq. (4) with measured displacement at the Vernier gauge and calculated average temperature of the microgauge. Fig. 13. shows calculated thermal expansion coefficient of poly-Si as a function of applied current. The measured thermal expansion coefficient of poly-Si was measured as constant, independent of applied current, in other words, the average temperature of the microgauge.

If the current was so small, in Fig. 13. 150uA, measured displacement was so small that the exact displacement was not measurable. If the current was large, the average temperature of the microgauge can exceed $200^{\circ} \mathrm{C}$. In this condition, the assumption in eq. (8) is no longer valid because we could not assure linear relationship of the resistivity characteristics. So the heat conduction equation was not always correct if the current was large. So the current was limited properly to avoid overheating above $200^{\circ} \mathrm{C}$. From some sets of measurements, we extracted that the thermal expansion coefficient of poly-Si thin film was $2.9 \times 10^{-6} / \mathrm{K}$ with standard deviation of $0.24 \times 10^{-6} / \mathrm{K}$. 


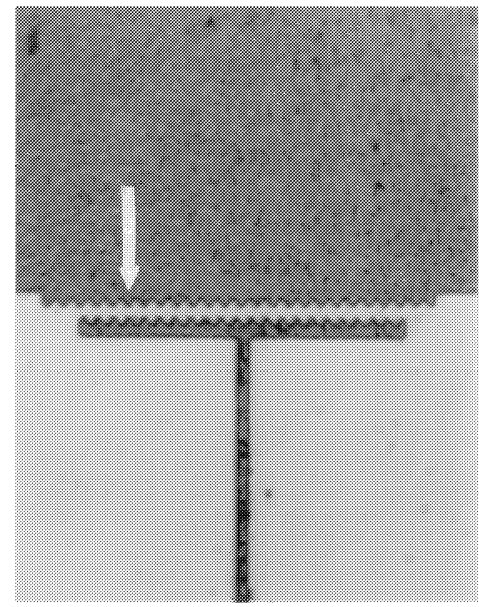

(a)

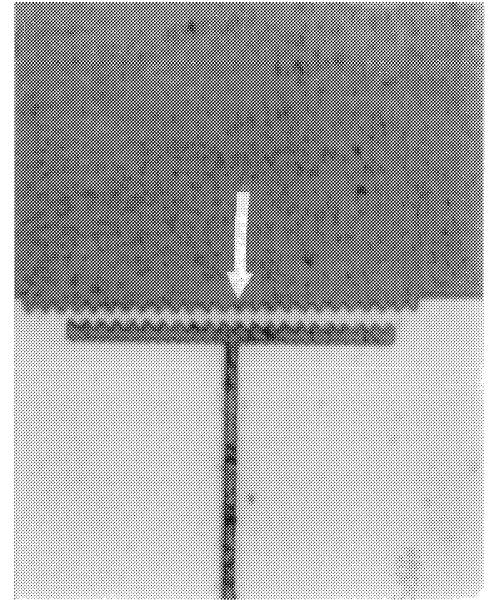

(b)

Fig. 12. CCD image of Vernier gauge of the microgauge.

(a) no current was applied

(b) current was applied.

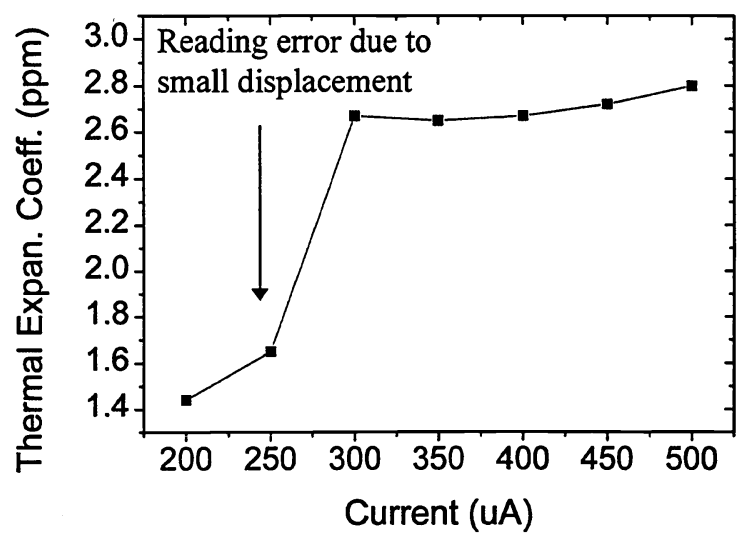

Fig. 13. Thermal expansion coefficient of poly-Si.

\section{CONCLUSION}

Thermal expansion coefficient of poly-Si thin film was measured by microgauge method. Microgauge method has some outstanding merits compared with commonly used optical methods. In the measurement process of thermal expansion coefficient of thin film, the Elastic modulus of the substrate and the thermal expansion coefficient of the substrate is no longer needed in microgauge method.

In the microgauge method, the thermal expansion coefficient of the thin film was expressed as a function of the average temperature of the microgauge and measured displacement at the Vernier gauge. When the current was applied to the microgauge, thermal expansion was produced by Joule heating and the average temperature of the microgauge rose. The amount of temperature rise was calculated from second order differential heat conduction equation. The amount of thermal expansion when current was applied was measured by Vernier gauge of the microgauge with CCD camera. From some sets 
of experiments, we extracted that the thermal expansion coefficient of poly-Si thin film is $2.9 \times 10^{-6} / \mathrm{K}$ with standard deviation of $0.24 \times 10^{-6} / \mathrm{K}$.

\section{ACKNOWLEDGMENT}

This project has been supported by the Support Projects of Fundamental Research in University 1997, by the Ministry of Information and Communication, Korea.

\section{REFERNECES}

1. Yao-Joe Yang and Chang-Jin Kim, Transducers, 337, 1995

2. J. Buhler, J. Funk, O. Paul, F. P. Steiner, H. Baltes, Sensors and Actuators, 46-47, 572, 1995

3. H. J. Ouenzer, A. Maciossek, B, Wagner, H. Pott, Transducers, 128, 1995

4. S. P. Murarka and T. F. Retajczyk. Jr., J. Appl. Phys., 54, 2069, 1983

5. T. F. Retajczyk. Jr., A. K. Sinha, Appl. Phys. Lett., 36, 161, 1980

6. Linwei Lin, Roger T. Howe and Albert P. Pisano, Proc. IEEE MEMS, 201, 1993

7. C. H. Mastrangelo, Ph. D. Dissertation, UC Berkeley, 1991 